
\title{
25 Research Square \\ Fatigue, Stigma, and Mood in Patients with Multiple Sclerosis: Effectiveness of Guided Imagery
}

\author{
Mina Beitollahi \\ Kerman University of Medical Sciences \\ Mansooreh Azizzadeh Forouzi \\ Kerman University of Medical Sciences \\ Batool Tirgari ( $\sim$ batool.tirgary@gmail.com ) \\ Kerman University of Medical Sciences

\section{Yunes Jahani} \\ Kerman University of Medical Sciences
}

\section{Research Article}

Keywords: Multiple sclerosis, Guided imagery, Fatigue, Stigma, Mood

Posted Date: December 13th, 2021

DOI: https://doi.org/10.21203/rs.3.rs-1059530/v1

License: (9) This work is licensed under a Creative Commons Attribution 4.0 International License. Read Full License 


\section{Abstract}

\section{Background and objectives}

: The present study aimed to assess the effectiveness of guided imagery on fatigue, stigma, and mood in patients with multiple sclerosis.

\section{Methods}

This clinical trial is a double-blind study that was conducted on 60 patients with multiple sclerosis referred to the largest center for special diseases in the southeast of Iran in 2020. The convenience sampling method was used to select the participants who were later divided into two groups of intervention $(n=30)$ and control $(n=30)$ using block randomization method. The intervention group listened to the guided imagery audio file at home for 25 minutes. The control group did not receive any intervention. Data were collected by demographic information questionnaires, Fatigue Severity Scale (FSS), Reece Stigma Scale for Multiple Sclerosis (RSS-MS), and the Profile of Mood States (POMS) before and one month after the intervention.

\section{Results}

According to the results, there was no significant difference between the two groups before the intervention in terms of the score of fatigue $(P<0.0=67)$, stigma $(P<0.64)$, and mood $(P<0.17)$. However, after the intervention, a significant differences was observed in this regard $(P<0.0001)$. In the intervention group, the mean score of fatigue decreased from $59.72 \pm 18.32$ to $35.8 \pm 16.15$, and the mean score of stigma decreased from $17.31 \pm 15.62$ to $5.09 \pm 8.06$, showing a significant reduction in the levels of fatigue $(P<0.0001)$ and stigma $(P<0.0001)$ compared to before intervention. Also, the mean score of mood decreased from $36.90 \pm 12.21$ to $28.55 \pm 11.87$, indicating an improvement in the mood of samples in the intervention group $(\mathrm{P}<0.0001)$.

\section{Conclusions}

The results indicated that guided imagery, as a cost-effective method, can decrease the fatigue and stigma, and enhance the mood of patients with MS. Therefore, nursing staff can use this method to improve MS patients' mood and decrease their fatigue and stigma.

\section{Introduction}

Multiple sclerosis is demyelinating, progressive, and inflammatory disease of the central nervous system (CNS) and the most common debilitating disease among young ${ }^{1}$ and middle-aged people ${ }^{2}$. On average, 
age of patients at the onset of MS ranges between 20 and 50 years ${ }^{3}$. The prevalence of MS is 6-200 per 100000 in the US and currently, around 400000 people have MS. Iran is considered to have an average level of prevalence, and 50000 Iranian people are affected by this disease ${ }^{1}$. The prevalence of MS in women is 2-3 times higher than in men ${ }^{3}$. Clinical progression of this disease negatively affects some aspects of life and psychological-behavioral function, and also leads to permanent, debilitation and unpredictable complications ${ }^{1}$. Complications of this disease include emotional and cognitive impairment, weakness, pain, depression, fatigue ${ }^{4}$, bowel-bladder dysfunction, sexual dysfunction ${ }^{3,4}$, balance problem ${ }^{1,4}$, social stigma ${ }^{1}$, and mood disorder ${ }^{3}$. Fatigue is the most common and earliest symptom of MS and affects $80 \%$ of affected people. Fatigue negatively affects people's function, daily activities, occupation, social activities, and quality of life ${ }^{5}$. Despite the use of pharmacological interventions for moderating the fatigue, side effects of the drugs and failure of these approaches have led to an increasing tendency toward complementary and alternative medicine (CAM) among MS patients ${ }^{6}$. Another complication of MS is stigma ${ }^{1,7}$, which was reported first by Goffman (1963), who defines stigma as a factor that makes people ignore or judge MS patients ${ }^{1}$. Moreover, stigma represents these patients as insignificant people in the eyes of others ${ }^{8}$. Approximately $72 \%$ of MS patients suffer from stigma ${ }^{1}$. Stigma has internal and external aspects; the external aspect relates to the negative and reprehensive views as well as unfair and discriminatory behaviors of others. The internal aspect refers to a feeling of being different from others that distorts patients' self-image, depression, and concealment of the disease $1,7,9$. Stigma and its consequences affect peoples' quality of life and leads to social isolation, loss of job and educational opportunities, unemployment, loss of self-confidence, depression, anxiety, and deprivation of social services $1,3,7,10$

Mood disorder is another symptom observed in MS patients. Mood disorder is defined as a change in people's attitudes that can lead to a change in social identity that negative effects psychological and mental health. The prevalence of mood disorder is high in MS patients, and it significantly affects their quality of life ${ }^{8}$.

Due to the unpredictability of MS, there is no definitive cure for this disease ${ }^{1}$, however, there are various methods that can reduce and moderate its symptoms ${ }^{3}$. Literature review indicates that nonpharmacological interventions such as physical, psychological, and cognitive interventions are effective in improving the symptoms of MS. Physical approaches include aerobic exercises, resistance training, electromagnetic field therapy, and cooling therapy. Psychological approaches include cognitive behavioral therapy, relaxation therapy, psychotherapy, energy conservation training, progressive muscle relaxation, and educational counseling ${ }^{5}$. The WHO's recommendation regarding complementary medicine is that people have a right to benefit from the least expensive, safest, and most effective medical interventions for the treatment of their diseases. Currently, in most developed countries, such as USA, England, France, and Germany, complementary medicine methods are being used along with other medical treatments ${ }^{11}$. The prevalence of using at least one of the complementary medicine treatments in one year has been reported at 56\% and $52.5 \%$ in European countries and in Iran's capital, respectively ${ }^{12}$. 
Guided imagery is one of the methods of complementary medicine ${ }^{2}$, which was used in the present study. Guided imagery is a mind-body technique, which intends to connect mind and body $2,6,13$. Considering the potential effects of immune system mediators, guided imagery uses guided images to treat immune system disorders such as MS by causing a change in the hypothalamic-pituitary-adrenal axis ${ }^{2}$. This medical intervention has many advantages; for instance, it improves quality of life, physical function, fatigue, pain, anxiety, depression, daily activities, and adherence to drug regimen 5 .

Different studies such as the study of Kaur et al. (2019) have shown that mental imagery is an innovative, effective, cost-effective, and convenient method that can be used as an independent or adjuvant method for treating neuropathic pain in MS patients, MI, stroke, and spinal cord injury ${ }^{14}$. Moreover, the study of Case et al. (2018) revealed that guided imagery has positive effects on MS patients' mood, fatigue, and quality of life ${ }^{15}$. Arseny et al. (2018) reported that people's ability to prevent, reduce, and ameliorate cognitive impairments has improved in MS patients by the advances made in pharmacological, neurological, psychological, and physical medicines, but there are still some deficiencies ${ }^{16}$. Chamanzari et al. (2013) conducted a study to assess the effects of guided imagery on the pain of orthopedic surgeries among traumatic patients referred to Hashemi Nezhad Hospital in Mashhad and their results indicated that guided imagery reduced pain and duration of pain, and also improved pain quality on the third day after the surgery in patients with fracture, while it did not affect pain intensity ${ }^{17}$. Jessica et al. (2020) conducted a study to assess the effect of hypnoanalysis and guided imagery on the ability of MS patients to identify and manage emotions and their results showed that, compared to hypnoanalysis, guided imagery intervention on a daily basis improved two subscales of quality of life in MS patients ${ }^{18}$. Therefore, there is a need for more studies to determine the effects of guided imagery on MS symptoms.

Employing complementary medicine in nursing is not new, and has been one of the interests of nursing theoreticians for a long time. However, nowadays, more attention is being paid to complementary medicine in the nursing profession due to changes in people's preferences. Ethical commitments, acting as strong stimuli, drive nurses toward using complementary medicine to moderate the problems of patients ${ }^{19}$. The present study is the first clinical trial to assess the effect of guided imagery on fatigue, stigma, and mood in MS patients in Iran.

\section{Methods}

\subsection{Population}

The present study is a double-blind clinical trial that was conducted on MS patients who were members of the MS society in the largest center for special diseases. Statistics Specialist and participants did not know which data are related to intervention or control group, and this showed that the study was doubleblinded. 
The sample size was calculated to be 70 patients ( $\mathrm{n}=35$ in each group), using the sample size formula and standard deviation extracted from the study of Case et al. (2018) with $90 \%$ power. Samples were selected by convenience method and based on the inclusion criteria. Using block randomization method with block size of 4 , and also $\mathrm{R}$ software, two participants were placed in each of the intervention and control groups within each block, and then, patients were randomly divided into control (35) and intervention (35) groups.

\subsection{Inclusion criteria}

\subsubsection{Participants}

Being 18-70 years old, having full consciousness, having auditory, visual, and verbal abilities, being able to read and write in Farsi, being diagnosed with relapsing-remitting MS by the neurologist, suffering from fatigue, and obtaining the score of less than 6 based on the FSS questionnaire were amongthe inclusion criteria.

\subsubsection{Intervention}

Patients were selected according to the inclusion criteria. The patients in the control group did not receive any intervention. However, patients in the intervention group in addition to their standard medication, listened to the guided imagery audio files for 20 minutes every day expect for Friday, for 12 sessions a week. The researcher held a briefing session for the intervention group and gave them the audio file. All the necessary explanations regarding listening to the guided imagery audio file were given to the patients. To reduce noises, after talking to patients and their families, a room was dedicated to the patients for 20 minutes to listen to the audio file. The patients listened to the audio file every week for 4 weeks in the morning (between 8:00 and 9:00) and evening (between 20:00 and 21:00) according to previous studies

20 . The transcript of the audio file included expressions for imagining desirable sceneries (forests, beaches, holy places...) along with proper effects and positive emphasis on reducing fatigue and stigma, and improving mood. The transcript was designed by the researcher based on Farsi and English sources and under the supervision of experts, which and finally was approved by the audio center of Kerman University of Medical Sciences. Patients were allowed to receive standard MS medication in both groups. During the study, in addition to sending reminder short messages, the participants were reminded to listen to the audio file and their possible questions were answered through phone calls.

\subsubsection{Types of trials}

The present study is a double-blind clinical trial.

\subsection{Exclusion criteria}

\subsubsection{Exclusion criteria included:}

Using sedative, Antidepressant or opioids, being absent for more than two sessions of the intervention, having a history of mental disease and continuous hospitalization, suffering from other nervous 
disorders, and having the experience of using relaxation methods (guided imagery).

\subsection{Data collection included:}

1. Demographic questionnaire that included questions about personal information such as age, gender, marital status, education, occupation, family's monthly income, and tobacco use, and medical information such as disease type (relapsing-remitting MS (RRMS), primary progressive MS (PPMS), secondary progressive MS (SPMS), and progressive-relapsing MS (PRMS)), as well as medication type, disease duration, number of relapses in the past year, and having other family members with MS. 2. The FSS questionnaire was used to assess the level of fatigue. This tool was designed by Krupp et al. (1989) and consists of 9 questions on 7-point Likert scale, ranging from 7 (the highest level of fatigue) and 1 (the lack of fatigue). To calculate the total score of fatigue, the scores attributed to each of the items were added and the sum was divided by 9 , this way the average score was obtained. A lower score indicated lower fatigue ${ }^{21,22}$. The validity and reliability of this questionnaire have been analyzed and confirmed in various studies. For instance, Farahani et al. (2012) investigated the reliability of the Iranian version of this tool in a sample of MS patients, and reported its internal consistency at 0.96 based on Cronbach's alpha ${ }^{23}$.

3. The RSS-MS questionnaire was used to assess stigma in patients. This tool was specially designed for the assessment of stigma in MS patients by Smith et al. (2019). It consists of 9 questions on 5-point Likert scale, ranging from 1 (never) to 5 (always). To measure the total score of stigma, the scores given to the items are added, and a higher score shows higher level of stigma ${ }^{12}$. The validity of this questionnaire has been confirmed by Smith et al. (2019), and its reliability has been reported to be 0.91 based on Cronbach's alpha.

4. POMS questionnaire was used to assess mood. This tool was designed by Nirola and Draplin in 1971. The validity and reliability of this tool have been evaluated in different studies such as those conducted by Albert et al. (2009) and Iwamitus et al. (2003). This questionnaire has 65 items and 6 subscales including anxiety/tension ( 9 items), depression/dejection (15 items), fatigue (7 items), confusion/bewilderment (12 items), anger/hostility (12 items), and ability/vigor (12 items). In this study, seven extra items were added to enrich the questionnaire, and therefore, they were not included in the scoring. The score ranges from zero to four based on the Likert scale. To calculate the total score of mood, the scores of 5 negative factors including anxiety, depression, anger, fatigue, and confusion are summed up and added and sum score of positive factor, which has been obtained by summing up the score of negative factors. Therefore, the total score of mood ranges from 0 to 168 , with the lower score indicating higher mood ${ }^{24}$. In Iran, this tool has been used by Tirgari et al. (2006) who reported an acceptable validity for it. They also reported its reliability to be 0.81 based on Cronbach's alpha.

\subsection{Statistics}

The collected data were entered into SPSS software version 22. Using dispersion and central indicators, independent sample t-test, paired t-test, Chi-Square, Fisher, and analysis of covariance (ANOVA), the data were analyzed at the significant level of 0.05 . 


\subsection{Ethics}

The present research was conducted after obtaining the code of ethics (IR.KMU.REC. 1398.263) from the Ethics Committee of Kerman University of Medical Sciences and Clinical trial code:

IRCT20151107024919N11 from Iranian Clinical Trial Center. After obtaining a written consent from patients, the first researcher explained the objective and method of study to MS patients, and emphasized on the principles of confidentiality and anonymity. The researcher also explained that the participation in the study is voluntarily, so participants can freely withdraw from the study at any time. In order to preserve the ethical issues, the audio file was provided to the patients in the control group at the end of the study, and also the results of the study were reported to the authorities and participants.

\section{Result}

Among the $70 \mathrm{MS}$ patients in this study, the data of 60 patients were analyzed, because 5 participants in the intervention group ( 2 women due to hospitalization for COVID-19 and 3 women due to not doing the assignments of more than two sessions) and 5 participants in the control group ( 1 woman and 1 man due to unwillingness to continue and 3 women due to family issues) were excluded from the study. Finally, data analysis was performed on 60 participants (30 participants in the intervention group and 30 in the control group).

According to the results, the mean age of participants was 39.73 years $( \pm 9.45)$ and 38.40 years $( \pm 10.29)$ in the intervention and control group, respectively, and the t-test did not reveal any significant difference between the two groups in terms of age $(P>0.05)$. Most participants in both groups were female, married, housewives, and had a high school diploma or higher education. There was no significant difference between the two groups in terms of demographic variables $(P>0.05)$, (Table 1$)$.

1- Fatigue: The mean score of fatigue before the implementation of guided imagery intervention was $57.97( \pm 13.33)$ and $59.72( \pm 18.32)$ in the control and intervention groups, respectively. Accordingly, there was no significant difference in the fatigue score between the two groups before the intervention $(P>0.05)$, (Table 2). However, the mean score of fatigue after the guided imagery intervention decreased by 23.64 in the intervention group. Therefore, it can be concluded that the level of fatigue was significantly different after the intervention $(P<0.0001)$, (Table 3).

2- Stigma: The mean stigma score before the guided imagery was $19.62( \pm 22.57)$ and $17.31( \pm 15.62)$ in the control and intervention groups, respectively. Accordingly, the scores of stigma were not significantly different between the two groups ( $P>0.05$ ), (Table 2). However, the mean score of stigma decreased by 5.72 in the intervention group, so that the level of stigma was significantly different after the intervention $(\mathrm{P}<0.0001)$, (Table 3).

3- Mood: The mean score of mood before the guided imagery was $41.21( \pm 12.58)$ and $37.39( \pm 8.28)$ in the control and intervention groups, respectively, so there was no significant different between the two groups $(P>0.05)$ in terms of the score of mood $(P<0.17)$, (Table 2$)$. However, the mean score of mood 
increased by 9.16 in the intervention group after the guided imagery intervention. As a result, the level of mood was significantly different after the intervention $(P<0.0001)$, (Table 3$)$.

\section{Discussion}

The results showed that guided imagery can significantly reduce fatigue and stigma, and also improve mood in MS patients. Mental imagery is a type of psychotherapy that works through auditory stimulation and includes some elements of cognitive-behavioral therapy. Guided imagery methods affect the hypothalamic-pituitary-adrenal axis. Case et al. (2018) investigated the effects of guided imagery on fatigue, mood, and quality of life of MS patients and their results showed that, this method is effective in reducing patients' fatigue and improving their mood and quality of life ${ }^{15}$. Although medical diagnoses in some studies have been different from that of the present study, these studies included chronic diseases, and all chronic diseases have some common features such as long-term complications of treatment, long-term use of medication and their side effects, frequent and lengthy hospitalization, fear of future, and sleeplessness, and patients are forced to live with the disease. Beizaee et al. (2017) examined the effects of guided imagery on hemodialysis patients, and showed that the intervention led to a decrease in patients' fatigue ${ }^{25}$. Buyukbayram et al. (2021) reported that guided imagery had a positive effect on cancer patients' fatigue ${ }^{26}$. The results of another study conducted by Lee et al. (2013) indicated that guided imagery led to a decrease in cancer patients' fatigue ${ }^{27}$. Fatigue in MS patients occurs due to damage done to the hypothalamus adrenal axis. So probably, the effects of guided imagery are exerted through relaxation and activation of damaged parts in the brain ${ }^{15}$.

Guided imagery uses human senses and based on the concept that the mind and body are connected, affects the body and psyche ${ }^{28}$. The literature review did not reveal any studies investigating the effect of guided imagery on stigma in MS patients. Lowther et al. (2018) assessed the effects of participating in palliative care intervention on HIV-associated stigma and their findings showed that patients' participation in the palliative care intervention increased their ability to overcome stigma, and improved their access to social positions and their image of self-identity and talents ${ }^{29}$. Toneti et al. (2019) believed that guided imagery creates psychological responses that interact with the hypothalamus axis and reduce stress, leading to a feeling of wellbeing and immune system compatibility. This relaxation method stimulates the areas responsible for creating desirable images and feelings in the brain by using visualization in a therapeutic and symbolic way ${ }^{30}$. Peters et al. (2015) proved that contact intervention led to a decrease in the stigma of patients affected by leprosy in Indonesia, and increased their awareness of the disease. Since guided imagery affects people's thoughts and imagination, it has been able to affect people's minds and repel the feeling of stigma through imagery ${ }^{31}$. Kolayiş $\mathrm{H}(2015)$ stated that guided imagery, in addition to improving the physical health, improves peoples' mental status; for instance, by controlling emotions and focus ${ }^{32}$. Moreover, guided imagery makes patients compatible with a large number of diseases ${ }^{33}$. Guided imagery reduces pain, stress, anxiety, and depression, and increases the feeling of well-being by increasing patient's focus ${ }^{25}$. Case et al. (2018) reported that guided 
imagery improved MS patients' mood ${ }^{15}$. Ensari et al. (2016) investigated the effect of single bouts of walking exercise and yoga on MS patients' mood, and their results indicated that walking had a positive effect on the patients' mood ${ }^{34}$. It is likely that in walking, besides physical stimulation, the body secrets some chemicals that change the mood. Moreover, the beautiful scenery and images that patients see while walking affect their mood. In guided imagery, patients are asked to imagine desirable images and sceneries such as flowers, plants, and sea, and use regular breathing exercises, which distance the mind from problems and create peace in patients, thereby improving their mood. Tsitsi et al. (2017) reported that relaxation intervention (progressive muscle relaxation and guided imagery techniques) had positive effects on anxiety and improved the mood of parents of hospitalized children with malignancies ${ }^{35}$. Shahabi et al. (2020) stated that in guided imagery, people are encouraged to take deep abdominal and diaphragmatic breaths and then release their muscles and imagine sceneries such as forests, beaches, and holy places, and pay attention to the smells and sounds around them. Studies have shown that focusing on positive visualization and imagery can relax and balance the mood. In fact, guided imagery increases resilience through distracting the mind from disturbing stimuli, relaxing patients, and affecting patients' mood ${ }^{36}$.

\subsection{Strengths and limitations}

The strengths of present study include; having more training sessions, having follow-up one month after the intervention, and being double-blind trial. On the other hand, weaknesses of this study include; lack of possibility of holding in-person and shared sessions due to COVID-19, which eliminated the synergic effect of shared sessions and the possibility of monitoring the intervention group, and the concurrence of sampling and COVID-19 pandemic.

Limitations of this study include; lack of possibility of monitoring the intervention at people's homes, modest sample size, the concurrence of sampling and COVID-19 pandemic, and use of convenience sampling. Therefore, it is recommended that in future studies on this issue, larger sample sizes should be used.

\section{Conclusion}

The results of present study indicated that guided imagery, as a non-invasive method of complementary medicine, has positive effects on reducing fatigue and stigma, and also improving the mood.

Guided imagery, as a non-invasive method of complementary medicine, can reduce the complications of MS. It is recommended that nursing managers improve the nursing staff's knowledge of no-invasive methods such as complementary medicine by arranging in-service training courses and informing them about the significance of these techniques in improving MS patients' fatigue, mood, and stigma. Guided imagery is a useful and cost-effective method that does not need special tools and moreover, does not cause any complications and is implementable in all situations. Therefore, the results of this study, in addition to being useful in nursing care, can be a basis for future studies. 


\section{Declarations}

\section{Acknowledgments}

The present study is the result of a MSc thesis in nursing at Kerman University of Medical Sciences with the code of research: 99000035 . The authors would like to express their deep appreciation to the patients who participated in this study. We would also like to offer our special thanks to the Honorable Vice Chancellor for Research of Kerman University of Medical Sciences that assisted authors in carrying out this research project.

\section{Ethics approval and consent to participate}

This study was approved by the Ethics Committee of Kerman University of Medical Science with the code of ethics No. (IR.KMU.REC. 1398.263). The clinical trial code (IRCT20151107024919N11) was received from the Iranian Clinical Trial Registration Center on 22/10/ 2020.

At the request of the Ethical committee, the study was conducted in accordance with the Declaration of Helsinki and Ethics Publication on Committee (COPE). Written informed consent was obtained from the patients. There was no ethical issue during the study and data collection. Furthermore, the participants were informed that they could withdraw from the study at any time. Moreover, they were ensured about confidentiality of information. Informed consent was obtained from all nurses. For the participations' information confidentiality to be ensured special codes were allocated to each questionnaire and the data were analyzed using these codes.

\section{Consent for publication}

Not applicable.

\section{Availability of data and materials}

The datasets used and/or analyzed during the current study are available and the corresponding author can deposit the data.

\section{Competing interests}

The authors declare that they have no competing interests.

\section{Funding}

This research received no financial support from any funding agency in the public, commercial, or not-forprofit sectors.

\section{Authors 'contributions}


$\mathrm{BT}, \mathrm{MB}$ and MAF contributed to conceiving and designing the research. The data were collected, analyzed, and interpreted by $\mathrm{BT}, \mathrm{MB}, \mathrm{MAF}$ and $\mathrm{YJ}$. BT, MB and MAF contributed equally to writing and revising the manuscript and approved the final manuscript.

\section{Acknowledgements}

The researchers appreciate all patients who spent their time so generously to participate in this study.

\section{Authors' information}

${ }^{1}$ School of Nursing and Midwifery, Kerman University of Medical Sciences, Kerman, Iran. ${ }^{2} \mathrm{PhD}$, Associate professor, Nursing Research Center, School of Nursing and midwifery. Kerman University of Medical Sciences, Kerman, Iran. ${ }^{3}$ Nursing Research Center, School of Nursing and midwifery. Kerman University of Medical Sciences, Kerman, Iran. ${ }^{4} \mathrm{PhD}$, Associate professor, Modelling in Health Research Center, Institute for Futures Studies in Health, Kerman University of Medical Sciences, Kerman, Iran.

\section{References}

1. Moshtagh M, Sajjadi H, Mowzooni H, Zeynalzadeh Ghoochani B. Authorized sclerosis and complementary care in Iran: A review study. Journal of Rehabilitation Medicine. 2017;6(1):234-253.

2. Ghazagh M, Zadhasan Z. The Effect of Group Mindfulness-Based Stress Reduction Program on the Quality of Life and Fatigue in Patients With Multiple Sclerosis. Avicenna Journal of Nursing and Midwifery Care. 2019;27(1):35-44.

3. Soleimani I, Taj al-Dini A. The effectiveness of cognitive-behavioral therapy based on fatigue on the dimensions of mental fatigue in patients with multiple sclerosis (MS): A case study. Clinical Psychology. 2016;8(2):13-21.

4. Motaharinezhad F, Parvaneh S, Ghahari S. Fatigue in people with multiple sclerosis: Cause, evaluation and treatment. Journal of Paramedical Sciences \& Rehabilitation. 2016;5(1):73-80.

5. Mohamadi M, Mohtashami J, Arab Khangholi Z. Stigma towards patients with mental disorders. Iranian Journal of Systematic Review in Medical Sciences. 2017;1(1):61-72.

6. Farzi S, Shamsaei F, Tapak L, Sadeghian E. Relationship Between the Stigma and the Family Performance of Psychiatric Patients. Iranian Journal of Nursing Research. 2020;14(6):1-8.

7. Ahmadnia S, Sharafat S, Kiana TK, Tavakoli C. The experience of stigma and the distorted identity of mothers with children with autism. Investigating Iran's social issues. 2017;8(1):103-117.

8. Tadayon Far Ma-R, Mohebbi M, Kooshan M, Rakhshani MH. The effect of guided visualization on the level of anxiety of patients under appendectomy surgery. Journal of Sabzevar University of Medical Sciences. 2014;20(5):681-688.

9. Afshar M, Spinani P. The effect of guided visualization on overt anxiety in cancer patients. Journal of Complementary Medicine, Arak School of Nursing and Midwifery. 2019;9(2):3718-3729. 
10. Tadayon Far Ma-R, Fuji S, Mohsenpour M, Rakhshani MH. Investigating the effect of sedation on the anxiety of patients under coronary angiography in Sabzevar Vasei Hospital in 2013. Journal of Sabzevar University of Medical Sciences. 2014;20(5):689-695.

11. Shahrukh Farahani A, Azimian M, Fallahpour M, Crimlo M. Validity and Reliability of the Persian Version of Multiple Sclerosis Fatigue Severity Scale Questionnaire. Rehabilitation. 2013;13(4):84-91.

12. Eldridge-Smith ED, Loew M, Stepleman LM. The adaptation and validation of a stigma measure for individuals with multiple sclerosis. Disability and rehabilitation. 2019:1-8.

13. Shafie Z, Babai S, Nazary A. The relationship between mood and quality of life in patients undergoing coronary artery bypass graft surgery. Iranian Journal of Nursing Research. 2013;26(83):57-67.

14. Kaur J, Ghosh S, Sahani AK, Sinha JK. Mental imagery training for treatment of central neuropathic pain: a narrative review. Acta Neurol Belg. 2019;119(2):175-186.

15. Case LK, Jackson P, Kinkel R, Mills PJ. Guided Imagery Improves Mood, Fatigue, and Quality of Life in Individuals With Multiple Sclerosis: An Exploratory Efficacy Trial of Healing Light Guided Imagery. J Evid Based Integr Med. 2018;23:2515690X17748744.

16. Sokolov AA, Grivaz P, Bove R. Cognitive Deficits in Multiple Sclerosis: Recent Advances in Treatment and Neurorehabilitation. Curr Treat Options Neurol. 2018;20(12):53.

17. Chamanzari $H$, Raghe S, Modarres Gharavi M, Ebrahimzadeh $S$. The effect of guided visualization on the pain of orthopedic surgery in trauma patients. Knowledge Horizon Quarterly. 2013;19(2):122118.

18. Brambila-Tapia AJL, Gutiérrez-García MM, Ruiz-Sandoval JL, et al. Using hypnoanalysis and guided imagery to identify and manage emotional aspects of multiple sclerosis $\{$ In press\}. EXPLORE. 2020:1-8.

19. Maryam A, Sedigheh G, Zinali M, Ahmadi N, Mirzaee S. The effectiveness of problem-solving skills on the quality of life and self-esteem of patients with MS. Quarterly Journal of Modern Psychological Ideas. 2017;1(1):29-38.

20. khalil nejhad a, zahed nasab h, khodabande lo h, et al. Diagnostic Biomarkers in Multiple Sclerosis. journal of ilam university of medical sciences. 2014;21(7):288-311.

21. Goldenberg MM. Multiple sclerosis review. Pharmacy and Therapeutics. 2012;37(3):175.

22. Brownlee WJ, Hardy TA, Fazekas F, Miller DH. Diagnosis of multiple sclerosis: progress and challenges. The Lancet. 2017;389(10076):1336-1346.

23. Howard J, Trevick S, Younger DS. Epidemiology of multiple sclerosis. Neurologic clinics. 2016;34(4):919-939.

24. Yaghoubi Nasr Abadi M, Atef Vahid MK, Ahmadzadeh GH. The effect of cognitive-behavioral group therapy on reducing depression and anxiety in patients with mood disorders. Iranian Journal of Psychiatry and Clinical Psychology. 2003;9(2):56-63. 
25. Beizaee Y, Rejeh N, Heravi Karimooi M, Tadrisi SD, Bahrami T. The Effect of Mind-guided Imagery on Decreasing Fatigue in Patients Undergoing Hemodialysis. Iranian Journal of Nursing Research. 2017;12(1):16-22.

26. Buyukbayram Z, Citlik Saritas $S$. The effect of Reiki and guided imagery intervention on pain and fatigue in oncology patients: A non-randomized controlled study. Explore (NY). 2021;17(1):22-26.

27. Lee MH, Kim DH, Yu HS. The effect of guided imagery on stress and fatigue in patients with thyroid cancer undergoing radioactive iodine therapy. Evid Based Complement Alternat Med. 2013;2013:130324.

28. Elgit Ö, Bilge A, Bayrakçı A. Effect of guided imagery on the functionality of individuals diagnosed with schizophrenia in a community mental health center. Journal of Psychiatric Nursing. 2020;11(3):165-172.

29. Lowther K, Harding R, Simms V, et al. Effect of participation in a randomised controlled trial of an integrated palliative care intervention on HIV-associated stigma. AIDS Care. 2018;30(9):1180-1188.

30. Toneti BF, Avelar JMP, Sousa FH, Toneti AN, Sonobe HM, Sawada NO. The meaning of integrative guided imagery relaxation therapy for women with breast cancer. Rev Esc Enferm USP. 2019;53:e03947.

31. Peters RM, Zweekhorst MB, Bunders JF, van Brakel WH. A cluster-randomized controlled intervention study to assess the effect of a contact intervention in reducing leprosy-related stigma in Indonesia. PLoS Negl Trop Dis. 2015;9(10):e0004003.

32. Kolayiş $\mathrm{H}$, îhsan $\mathrm{S}$, Özge $\mathrm{K}$. Takım sporlarıyla uğraşan kadın sporcuların imgeleme, güdülenme ve kaygı puanları arasındaki ilişkinin incelenmesi. Spormetre Beden Eğitimi Ve Spor Bilimleri Dergisi. 2015;13(2):129-136.

33. Santos A. Nurse's guide to guided imagery. Nursing2020. 2016;46(1):55-58.

34. Ensari I, Sandroff BM, Motl RW. Effects of Single Bouts of Walking Exercise and Yoga on Acute Mood Symptoms in People with Multiple Sclerosis. Int J MS Care. 2016;18(1):1-8.

35. Tsitsi T, Charalambous A, Papastavrou E, Raftopoulos V. Effectiveness of a relaxation intervention (progressive muscle relaxation and guided imagery techniques) to reduce anxiety and improve mood of parents of hospitalized children with malignancies: A randomized controlled trial in Republic of Cyprus and Greece. Eur J Oncol Nurs. 2017;26:9-18.

36. Shahabi A, Ehteshamzadeh P, Asgari P, Makvandi B. Comparison of the Effectiveness of Acceptance and Commitment Therapy and Guided Imagery on the Resilience of Cardiac Disease Patients Referring to the Heart Rehabilitation Department. The Horizon of Medical Sciences. 2020;26(3):276297.

\section{Tables}

Table 1: Comparison of Demographic variables between groups 


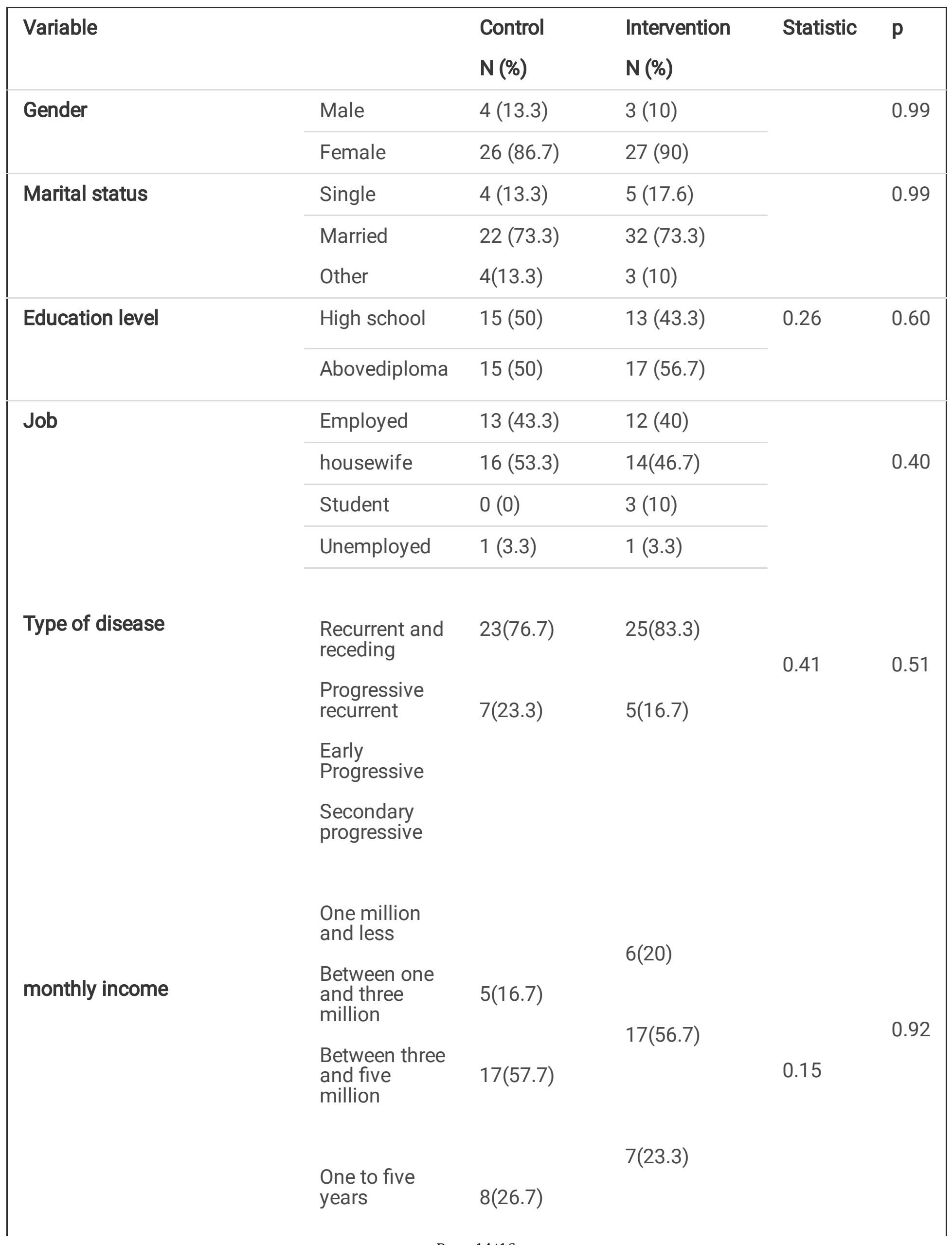


Five to ten

years

Ten years and

older

13(43.3)

Time of onset of the disease $\quad$ Once or twice 11(36.7)

More than

twice

$8(26.7)$

$11(36.7)$

Interferon

$9(30)$

0.69

beta

$8(28.7)$

Recurrence in

Glatiramer

acetate

28(93.3)

One year ago

Fingolimod

26(86.7)

Rituxymab

2(13.3)

Diphosel

$4(6 / 7)$

Other

Type of drug used

None

8(26.7)

13(43.3) 4(13.3)

1(3.3)

Yes

3(10)

No

Yes

$5(16.7)$

3(10)

1(3.3)

8(26.7)

drug use

No

5(16.7)

$0(0)$

3(10)

4(13.3)

2(6.7)

$0(0)$

$0(0)$

30(100)

Family history

$\begin{array}{ll}9(30) & 26(86.7) \\ 21(70) & \end{array}$


Table 2: Comparison of Fatigue, Stigma and Mood between two groups before Intervention

\begin{tabular}{|lllllll|}
\hline Variable & Group & Mean & SD & $\mathbf{t}$ & $\mathbf{d f}$ & $\mathbf{p}$ \\
\hline Fatigue & Control & 57.97 & 13.33 & 0.42 & 58 & 0.67 \\
\cline { 2 - 5 } & Intervention & 59.72 & 18.32 & & & \\
\cline { 1 - 5 } Stigma & Control & 19.62 & 22.57 & 0.46 & 58 & 0.64 \\
\cline { 2 - 4 } & Intervention & 17.31 & 15.62 & & & \\
Mood & Control & 41.21 & 12.58 & 1.38 & 50.16 & 0.17 \\
& Intervention & 37.39 & 8.28 & & & \\
\cline { 2 - 4 } & & & & & & \\
\hline
\end{tabular}

Table 3: Comparison of Fatigue, Stigma and Mood between two groups after Intervention

\begin{tabular}{|c|c|c|c|c|c|}
\hline Variable & Group & Mean $\pm S D$ & Mean difference & $\mathbf{F}$ & p \\
\hline \multirow[b]{2}{*}{ Fatigue } & Control & $58.42 \pm 5.68$ & \multirow[b]{2}{*}{ 23.64(53.30.75.16) } & \multirow[b]{2}{*}{27.47} & \multirow[b]{2}{*}{$<0.0001$} \\
\hline & Intervention & $78.34 \pm 5.68$ & & & \\
\hline \multirow{2}{*}{ Stigma } & Control & $05.16 \pm 1.73$ & \multirow{2}{*}{ 5.72(10.63.0.83) } & \multirow{2}{*}{5.43} & \multirow{2}{*}{0.023} \\
\hline & Intervention & $33.10 \pm 1.73$ & & & \\
\hline \multirow{3}{*}{ Mood } & Control & $63.29 \pm 3.29$ & \multirow{3}{*}{$9.16(5.13 .13 .24)$} & \multirow{3}{*}{20.65} & \multirow{3}{*}{$<0.0001$} \\
\hline & & & & & \\
\hline & Intervention & $79.38 \pm 3.29$ & & & \\
\hline
\end{tabular}

